\title{
Case Report and Review of the Literature
}

\section{Polymicrobial Infection as a Rare Cause of Osteomyelitis: Case Presentation and Review of the Literature}

\author{
Mohs $E^{*}$, Darwich A, Dally F, Assaf E and Gravius $S$ \\ Orthopaedic and Trauma Surgery Centre, University Hospital Mannheim, Medical Faculty Mannheim of the University of Heidelberg, Mannheim, \\ Germany

\section{A R T ICLE INFO} \\ Article history: \\ Received: 9 February, 2021 \\ Accepted: 23 February, 2021 \\ Published: 22 March, 2021 \\ Keywords: \\ Osteomyelitis \\ polymicrobial \\ femur

\begin{abstract}
A B S T R AC T
This case report presents a 48-year-old patient with suspicion for chronic osteomyelitis caused by oral pathogens including species of Actinomyces, Eikenella and Stenotrophomonas. The symptoms included progressive swelling of the right thigh and hyperpyrexia with up to $41^{\circ} \mathrm{C}$. Imaging methods included standard X-ray as well as MRI. Furthermore, two operations were necessary for surgical infection therapy. Histological and microbiological investigations of intraoperative samples showed polymicrobial osteomyelitis. Since local trauma could be excluded as the cause for the disease, the patient's dental status was evaluated and proved as the possible infectious focus with a hematogenous spread. The patient was treated with intravenous antibiotics for two weeks and oral antibiotics for four further weeks. The patient's clinical condition drastically improved with that treatment.
\end{abstract}

\section{Introduction}

In this case, we describe to our knowledge, the first report of chronic osteomyelitis of the proximal femur caused by hematogenous polymicrobial infections by pathogens of the human oral flora. Chronic hematogenous osteomyelitis of the femur in immunocompetent adult patients caused by pathogens of the oral flora seems to be an extremely rare disease, but this cause needs to be taken into consideration in patients presenting with a long-lasting pain. Osteolytic lesions should be taken very seriously as this condition is often missed or delayed diagnosed and the associated complications of such a delay can be very drastic. In three publications, patients presented with pathological fractures, had a missed osteomyelitis [1].

\section{Case Presentation}

A 48-year-old Pakistani female patient presented to our emergency department as a transfer from another hospital with progressive swelling of the right thigh. She has not been in her home country for years. Except for a NPP in the cervical spine $5 / 6$, no preexisting diseases were known. The patient presented with hyperpyrexia with up to $41^{\circ} \mathrm{C}$ as well as highly elevated inflammatory parameters (CRP $180 \mathrm{mg} / \mathrm{l}$, Leukocytes $\left.>18,0 \times 10^{\wedge} 9 / 1\right)$. The first clinical examination showed a charmless skin without any scars but subtle warming of the right thigh without reddening. She did not show any sensorimotor deficits. The movement of the hip was not limited. Initially, an X-ray picture of the right femur and the pelvis was done, which showed cortical thickening and suspicion for abscess formation or for osteosarcoma with the formation of osteolysis (Figure 1). For better evaluation, an MRI of the right hip joint, the femur and the pelvis was performed, showing a pronounced fusing soft tissue process at the right femur with an intraosseous communication. It is also highly suspicious for an abscess formation and accompanying osteomyelitis (Figure 2). For completion of the imaging, an ultrasound of the soft tissue of the right tight was done showing fluctuating lesions and fluid accumulation in the soft tissue.

${ }^{*}$ Correspondence to: Mohs Elisabeth, Orthopaedic and Trauma Surgery Centre, University Hospital Mannheim, Medical Faculty Mannheim of the University of Heidelberg, Theodor-Kutzer-Ufer 1-3, 68165, Mannheim, Germany; Tel: +4917629962346; E-mail: Elisabeth.Mohs@umm.de 

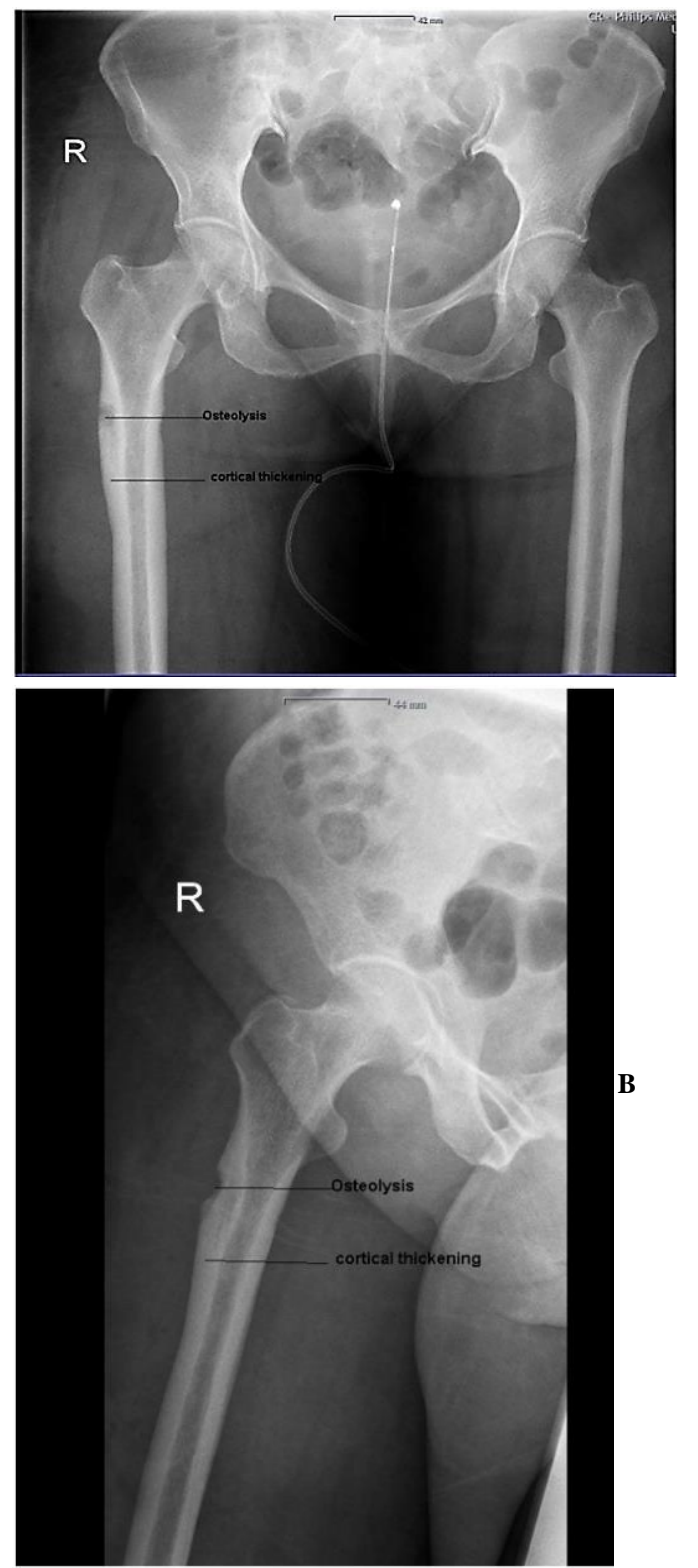

Figure 1: A) AP X-ray of the pelvis and B) Lauenstein's view of the right hip showing thickening of the lateral corticalis on the right femur and suspicion for abscess formation or for osteosarcoma with the formation of osteolysis. The thickening of the lateral corticalis can be especially seen distal to the greater trochanter laterally. Here, also a zone of osteolysis can be identified.

Urgent debridement of the confluent abscess formations in the soft tissues and of the femur was undertaken through a lateral approach to the thigh. Intraoperatively, a fistula was found, which releases bloody putrid cell debris indicating abscess formation. Biopsies were also sent for microbiological and histopathological examination. An empiric antibiotic therapy with Ceftriaxone $1 \mathrm{~g} 2$ times a day and Flucloxacillin $2 \mathrm{~g}$ six times a day was started. Two days later, the microbiology results showed the growth of Actinomyces israelii and Eikenella corrodens, which are both pathogens of the oral flora of humans and animals. The medical history regarding a potential transmission of these pathogens, i.e. a bite injury to humans or animals, showed no evidence. Prolonged culture and histological examination demonstrated subacute osteomyelitis with soft tissue abscess formation with no signs of malignant disease. After consulting our microbiologist, the antibiotic therapy was switched to Ampicillin 2g three times daily according to the antibiogram.
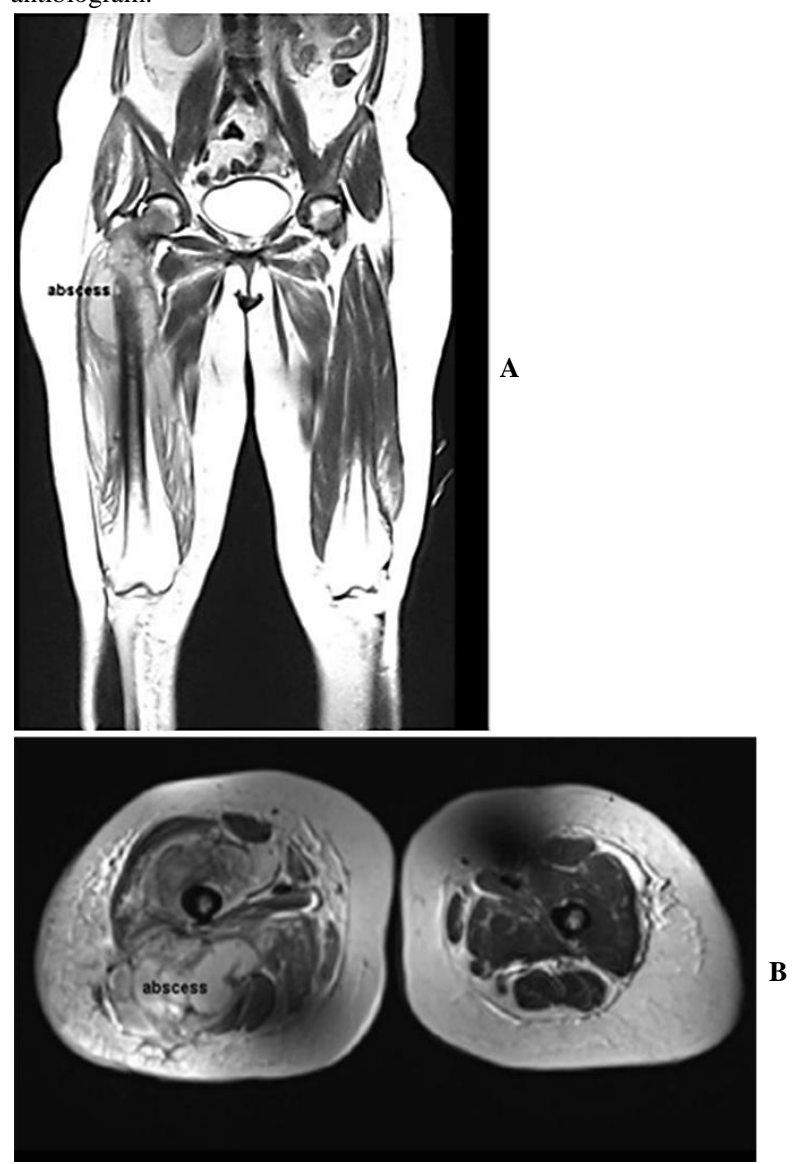

Figure 2: MRI sections A) sagittal and B) coronal showing the right femur and the soft tissue surrounding it. An abscess formation in the lateral thigh can be identified as well.

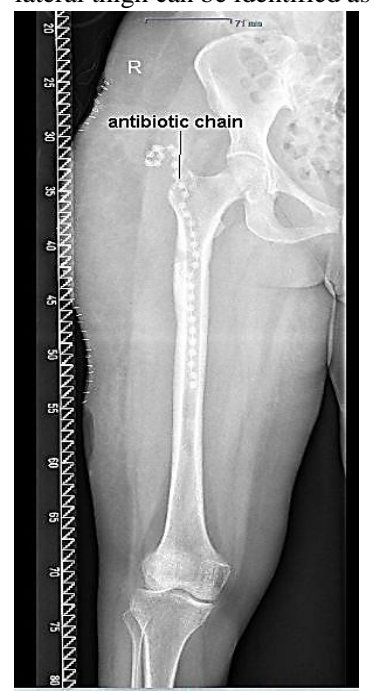

Figure 3: Postoperative AP X-ray of the right femur after the second operation with opening and drilling of the femur medullary cavity and insertion of the antibiotic chain as well as removal of osteolytic lesions in the lateral cortex. Enclosed the antibiotic chain inside the proximal medullary cavity for local intramedullary therapy. 
Three weeks later, a second operation had to be performed around three weeks later due to persistent pus secretion of the wound. Intraoperatively, the femur was relieved by drilling and the surrounding necrotic tissue was debrided. Drilling the bone released a large amount of putrid secretion. The fistula formation extending from the bonemarrow space through the soft tissue to the outer skin was explored and excised. The vastus lateralis muscle showed multiple areas of necrosis, which were excised, too. Furthermore, gentamycin secreting Palacos ${ }^{\circledR}$ (Fa. Heraeus Medical GmbH, Wehrheim, Germany) chain was used to treat the infection locally (Figure 3). Again, biopsies for the histological and microbiological examination were sent. The microbiological investigation demonstrated another pathogen, Stenotrophomonas maltophilia, which is also a pathogen of the oral flora. Therefore, the antibiotic therapy was changed again to Cotrimoxazole $960 \mathrm{mg}$ three times a day and Unacid $3 \mathrm{~g}$ four times a day. To identify possible spreading sources, an X-ray of the jaw was done to evaluate the patient's dental status showing pathologies, especially in the maxilla. These pathologies were malpositioning and degeneration of the teeth roots (Figure 4).

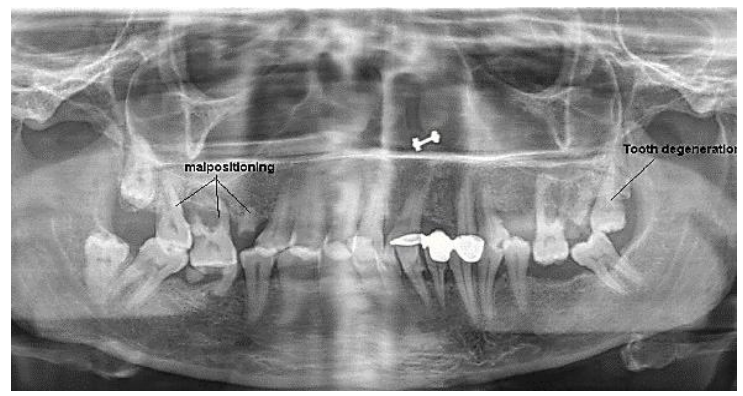

Figure 4: X-ray of the jaw and dental status of the patient showing malalignment and root degeneration, especially in the molars. In the maxilla, the patients' piercing is visible at the incisor teeth. In the mandible, tooth crowns can be identified at the left lateral incisor and the left canine tooth.

Also, X-ray of the chest was performed, showing no pathologies for suspicion of hematogenous spread. The urine was examined as well, showing no abnormalities, such as urinary tract infections. Under antibiotic therapy, the patient recovered quite quickly, she was able to mobilize herself and the insertion wound was dry without any signs of infection. The antibiotic chain was left inside. After few months, the antibiotic chain was removed. In follow-up sessions, the patient presented clinically without any symptoms, the scar formed after the operative incision was without any signs of infection.

\section{Discussion}

Osteomyelitis, in general, is a well-known medical condition usually occurring due to spread through adjacent infected tissue or in association with an open fracture. Spontaneous hematogenous osteomyelitis is a rare form that was described in only a few reports in the literature so far [14]. Osteomyelitis can be classified according to time of onset in acute arising within days to weeks and chronic occurring over months to years. The chronic form is usually associated with avascular bone necrosis and the formation of sequesters. In adults, both acute and chronic forms are most commonly caused by Staphylococcus aureus. Other common pathogens include coagulase-negative Staphylococci and Streptococci [5]. Osteomyelitis can also be classified according to the cause into endogenous and exogenous. Exogenous osteomyelitis occurs due to trauma, surgery or insertion of foreign material (for example, a total joint replacement (TJR)). On the other side, endogenous osteomyelitis is due to vascular insufficiency, i.e. in diabetic foot patients or due to hematogenous spread.

The present case describes an atypical polymicrobial osteomyelitis caused by oral pathogens including species of Actinomyces, Eikenella and Stenotrophomonas. To our knowledge, such a case was described in only a few English and German medical literature publications, for instance, by Dong JR et al., who described Actinomycotic osteomyelitis in an immunocompromised adult [6]. Osteomyelitis of a long bone in an immunocompetent adult, caused by Actinomyces as a monomicrobial infection, was already described by Dong Jin Ryo et al. in 2019, but our patient showed polymicrobial growth of pathogens in the osteomyelitic lesion [6]. A hematogenous (endogenous) spread from the oral cavity was assumed to be the cause of osteomyelitis. In accordance with our assumption, pathological changes in some tooth roots were found in the upper jaw. The medical history and clinical examination showed no signs of local trauma or surgery as a cause of exogenous osteomyelitis typically associated with a polymicrobial infection.

Endogenous osteomyelitis, as a result of a hematogenous spread after bacteremia, is usually a monobacterial infection [5]. The most common pathogen for endogenous and exogenous osteomyelitis is Staphylococcus aureus, which can be found on the skin, in nostrils, perineum, colon and vagina of humans. The pathogens found in this case were not quite typical for causing osteomyelitis but can be found in the human oral flora. Actinomyces and Eikenella are physiologically present in the oral flora, while Stenotrophomonas can be found in urine and respiratory tract and oral flora as well. Eikenella corrodens is a gramnegative, microaerophilic bacillus and only lately got into attention as a possible pathogen for osteomyelitis. Up to now, only a local infection with Eikenella corrodens, for instance, by bite wounds, was known to cause osteomyelitis in humans, as described by Johnson et al. [7]. The management, in this case, included local surgical debridement as well as a pathogen-specific antibiotic therapy. In our case, a local infection due to a bite wound could be ruled out by medical history and clinical examination.

Also, Stenotrophomonas maltophilia is only known as a rare cause of osteomyelitis. The pathogen can be found ubiquitary in soil, but also in human urine and respiratory tract secretions as well as in the oral flora [8]. It is a gram-negative rod so far only known for causing local osteomyelitis [9]. It also often causes infection of endotracheal and tracheostomy tubes. Especially in immunocompromised patients, Stenotrophomonas can also cause nosocomial infections. The third pathogen, Actinomyces israelii is an oral pathogen causing osteomyelitis in the jawbone, particularly in the maxilla and is an extremely rare disease [10]. The typical clinical picture is one of abscesses opens draining sinuses [10]. Actinomyces can also cause osteomyelitis of the sternum [11].

In summary, the pathogens found in this patient, Eikenella, Stenotrophomonas and Actinomyces, can cause acute osteomyelitis, usually of the mandible [8-10]. Chronic osteomyelitis due to these pathogens localized in the proximal femur is so far unknown in literature. Thein et al. investigated in their work in 2013 three cases of 
hematogenous femur osteomyelitis caused by unusual bacteria. All three cases were caused by Streptococcus anginosus, Staphylococcus intermedius and Aggregatibacter aphrophilus. Here also no evidence of the bacteria found in our patient. In general, osteomyelitis needs to be treated for a long time to be cured. Treatment of osteomyelitis, in general, includes surgical debridement and proper antibiotic therapy [12]. The patient was operated twice and the pathogen-specific antibiotic therapy was given intravenously for 14 days after the second operation. She was then given an oral antibiotic directed to the pathogens for four more weeks. It should be noted that two operations were necessary for successful treatment. The third pathogen, Stenotrophomonas, was detected during the second operation. Here, we assume that the antibiotic therapy initiated after the first surgery was insufficient since it did not cover the third pathogen revealed after the second surgical procedure. A possible cause can be the difficult cultivation of the pathogens in culture. Actinomyces and Eikenella can be detected in the laboratory on agar plates but take about two weeks to grow. Stenotrophomonas can be grown on MacConkey agar plates and needs a prolonged culture to be detected.

In conclusion, in this case, we describe to our knowledge, the first report of chronic osteomyelitis of the proximal femur caused by hematogenous polymicrobial infections by pathogens of the human oral flora. Chronic hematogenous osteomyelitis of the femur in immunocompetent adult patients caused by pathogens of the oral flora seems to be an extremely rare disease, but this cause needs to be taken into consideration in patients presenting with a long-lasting pain. Osteolytic lesions should be taken very seriously as this condition is often missed or delayed diagnosed and the associated complications of such a delay can be very drastic. In three publications, patients presented with pathological fractures, had a missed osteomyelitis [1].

Since there was no suspicion for a local entry gate, such as a local trauma or a bite injury, we suspected a hematogenous spread from the oral cavity to be the underlying factor of the disease. This suspicion was confirmed in the performed radiological investigations. The treatment with surgical debridement and local and systemic antibiotics showed a satisfying infection control.

\section{Ethical Approval}

All procedures performed in studies involving human participants were in accordance with the ethical standards of the institutional and/or national research committee and with the 1964 Helsinki declaration and its later amendments or comparable ethical standards. Formal consent is not required for this type of study.

\section{Consent for Publication}

Not applicable.

\section{Availability of Data and Materials}

Not applicable.

\section{Competing Interests}

The authors declare that they have no known competing financial interests or personal relationships that could have appeared to influence the work reported in this paper.

\section{Funding}

None.

\section{Author Contributions}

Mohs E and Gravius S designed and directed the project of the case report as well as supervised the patient during surgery and stationary stay. All authors discussed the results and commented on the manuscript. Mohs E wrote the paper with input from all authors.

\section{Acknowledgment}

Not applicable.

\section{REFERENCES}

1. Thein R, Tenenbaum S, Chechick O, Leshem E, Chechik A et al. (2013) Delay in diagnosis of femoral hematogenous osteomyelitis in adults: an elusive disease with poor outcome. Isr Med Assoc J 15: 85-88. [Crossref]

2. Roberts DE (1998) Femoral osteomyelitis after tooth extraction. Am J Orthop (Belle Mead NJ) 27: 624-626. [Crossref]

3. Seybold U, Talati NJ, Kizilbash Q, Shah M, Blumberg HM, Franco Paredes C (2007) Hematogenous osteomyelitis mimicking osteosarcoma due to community associated methicillin-resistant staphylococcus aureus. Infection 35: 190-193. [Crossref]

4. Zalavras CG, Rigopoulos N, Lee J, Learch T, Patzakis MJ (2009) Magnetic resonance imaging findings in hematogenous osteomyelitis of the hip in adults. Clin Orthop Relat Res 467: 1688-1692. [Crossref]

5. Jason HC, Manring MM, Shirtliff M et al. (2009) Osteomyelitis of the Long Bone. Semi Plast Surg 23: 59-72. [Crossref]

6. Dong JR, Jeon YS, Kwon HY, Choi SJ, Roh TH et al. (2019) Actinomycotic Osteomyelitis of a long bone in an immunocompetent adult: a case report and literature review. BMC Musculoskeletal Disord 20: 185. [Crossref]

7. Johnson SM, Pankey GA (1976) Eikenella corrodens osteomyelitis, arthritis and cellulitis of hand. South Med J 69: 535-539. [Crossref]

8. Linke J, et al. (2004) Implantatversorgung einer Patientin nach Lebertransplantation-klinische und mikrobiologische Langzeitergebnisse. Deutscher Ärzteverlag Köln, Zahnärztliche Impl 20: 223-227.

9. Chesnutis EJ, Ng A, Kruse D, Stone PA (2018) Stenotrophomonas maltophilia: A rare Case of Osteomyelitis after an open distal tibia fracture. J Foot Ankle Surg 57: 1037-1041. [Crossref]

10. Bahar S, Akdeniz BG, Günbay S, Hilmioğlu Polat S, Başdemir G (2017) Actinomycosis osteomyelitis of the jaws: Report of four cases and a review of the literature. J Dent Sci 3: 301-307. [Crossref]

11. Lee JH, Jeon SC, Jang HJ, Kim H, Kim YH et al. (2015) Primary Sternal Osteomyelitis Caused by Actinomyces israelii. Korean J Thorac Cardiovasc Surg 48: 86-89. [Crossref] 
12. Walter G, Kemmerer M, Kappler C, Hoffmann R (2012) Treatment Algorithms for Chronic Osteomyelitis. Deutsch Ärztebl Int 14: 257264. [Crossref] 